\title{
Direct sampling of exponential phase moments of smoothed Wigner functions
}

\author{
Jaromír Fiurášek \\ Department of Chemical Physics, The Weizmann Institute of Science, Rehovot 76100, Israel
}

(October 29, 2018)

We investigate exponential phase moments of the $s$ parametrized quasidistributions (smoothed Wigner functions). We show that the knowledge of these moments as functions of $s$ provides, together with photon-number statistics, a complete description of the quantum state. We demonstrate that the exponential phase moments can be directly sampled from the data recorded in balanced homodyne detection and we present simple expressions for the sampling kernels. The phase moments are Fourier coefficients of phase distributions obtained from the quasidistributions via integration over the radial variable in polar coordinates. We performed Monte Carlo simulations of the homodyne detection and we demonstrate the feasibility of direct sampling of the moments and subsequent reconstruction of the phase distribution.

PACS number(s): 42.50.Dv, 03.65.Bz

\section{INTRODUCTION}

Quantum-state tomography is a powerful tool allowing us to reconstruct the quantum state of a traveling optical mode, provided that many identical copies of the state can be prepared [1]:2]. The idea of homodyne tomography stimulated research in the field of quantum-state reconstruction of other simple quantum-mechanical systems. Recently, reconstructions of the quantum state of a molecular vibrational mode [3] and the motional quantum state of a trapped ion [4,5 have been reported.

Optical homodyne tomography relies on balanced homodyne detection. The signal field is mixed with a strong coherent local oscillator (LO) at a lossless 50/50 beam splitter. Both the LO and the signal are derived from a common master oscillator to ensure a stable phase difference $\theta$ between them. Two photodetectors are placed at the output ports of the beam splitter and the measured photocurrents are subtracted. The resulting signal is proportional to the rotated quadrature of the signal mode $x_{\theta}$. The measurement, which yields the probability distribution $w\left(x_{\theta}, \theta\right)$ of the quadrature $x_{\theta}$, is repeated for many different phase shifts $\theta$ from interval $[0,2 \pi]$.

The Wigner function of the signal mode can be recovered from the measured statistics $w\left(x_{\theta}, \theta\right)$ by means of inverse Radon transform [6,1]. Numerical implementation of this inversion is not simple and a filtering algorithm has to be applied to achieve the desired reconstruction. To avoid these complications, it was suggested to directly get quantities of interest from the measured data by averaging appropriate kernels over the distributions $w\left(x_{\theta}, \theta\right)$. This approach proved to be very fruitful, and kernels for the direct sampling of density-matrix elements in the Fock basis $\rho_{m n}$ []], the moments $\left\langle a^{\dagger j} a^{k}\right\rangle$ [8], Fourier coefficients of the canonical phase distribution [9], and for smoothed Wigner functions [10] have been found. A different approach to the quantum-state reconstruction employs a maximum likelihood estimation [11. It was demonstrated recently that this technique can be used to estimate photon number distribution 12 and even a whole density matrix $[13$. For a review, see |14.

In recent years, great attention has been devoted to the quantum phase. Canonical phase distribution introduced by London 15 represents a limit of Pegg-Barnett phase formalism 16]. Recently, an approximate measurement of the canonical phase distribution, using the phase-coherent states, has been proposed [17]. One can also construct phase distributions from the phase-space quasidistributions 18 20. The phase distribution obtained from the $Q$ function (or smoothed $Q$ function in the case of imperfect detection) can be directly measured 21,22. An operational approach to the quantum phase, based on the description of a given experimental setup, has been proposed by Noh et al. 23. The relation between canonical and measured phase distributions was discussed in 24. For a recent review, see 20,25.

Canonical phase distribution as well as phase distributions obtained from quasidistributions cannot be directly sampled from the homodyne data. One has to reconstruct the Wigner function or the density matrix and then use the definition of the phase distribution to calculate it 26]. This detour via the Wigner function or the density matrix complicates numerical data processing and increases error in the final result. However, the exponential phase moments (Fourier coefficients) of the canonical phase distribution can be directly sampled with the use of appropriate kernels [9]. Phase-number uncertainty relations can be verified by sampling the first exponential moment of the canonical phase distribution and the photon-number variance 27. It was also pointed out in [9] that the exponential phase moments of the Wigner function can be directly sampled.

But we do not have to restrict ourselves to the exponential phase moments of canonical phase distribution or the Wigner function. In this paper, we consider direct sampling of the exponential phase moments of general $s$ parametrized phase distributions. We show that it is possible to directly sample the exponential phase moments of any $s$-parametrized quasidistribution for $s<-(1-\eta) / \eta$, 
where $\eta$ is the overall detection efficiency. Namely, we find the expressions for the kernels whose average over data recorded in balanced homodyne detection yields the exponential phase moments. We show that a knowledge of these moments as functions of $s$ and the photonnumber distribution provides complete characteristics of a given quantum state. The phase moments are Fourier coefficients of the phase distributions defined as radial integrals of the $s$-parametrized quasidistributions in the polar coordinates. We demonstrate that these phase distributions can be successfully reconstructed from the sampled phase moments.

The paper is organized as follows. In Sec. II the exponential phase moments are introduced and discussed. In Sec. III simple analytical expressions for the sampling kernels are derived and the influence of imperfect detection is addressed. In Sec. IV the results of Monte Carlo simulations are presented. Section V contains conclusions. Some mathematical issues are linked to the Appendix.

\section{EXPONENTIAL PHASE MOMENTS}

The quasidistributions related to various $s$ orderings of creation and annihilation operators can be expressed in terms of the density matrix $\rho$ [28],

$$
W(\alpha, s)=\frac{1}{\pi^{2}} \int e^{s|\beta|^{2} / 2} \operatorname{Tr}\left[\rho e^{\left(a^{\dagger}-\alpha^{*}\right) \beta-(a-\alpha) \beta^{*}}\right] d^{2} \beta,
$$

where $a, a^{\dagger}$ are annihilation and creation operators. One gets the $P$ representation for $s=1$, the Wigner function for $s=0$ and the $Q$ function for $s=-1$. The $s$-parametrized quasidistributions are mutually related through the convolution

$$
\begin{aligned}
W\left(q, p, s_{2}\right)= & \frac{1}{\pi\left(s_{1}-s_{2}\right)} \\
& \times \int_{-\infty}^{\infty} \int_{-\infty}^{\infty} \exp \left[-\frac{\left(q-q^{\prime}\right)^{2}+\left(p-p^{\prime}\right)^{2}}{s_{1}-s_{2}}\right] \\
& \times W\left(q^{\prime}, p^{\prime}, s_{1}\right) d q^{\prime} d p^{\prime}
\end{aligned}
$$

where $q=\left(\alpha+\alpha^{*}\right) / \sqrt{2}$ and $p=-i\left(\alpha-\alpha^{*}\right) / \sqrt{2}$ are the usual quadratures, and $s_{1}>s_{2}$ must hold.

It is convenient to introduce polar coordinates $q=$ $r \cos \theta, p=r \sin \theta$. The phase distribution $P_{s}(\theta)$ related to $s$-parametrized quasidistribution is defined as [20]

$$
P_{s}(\theta)=\int_{0}^{\infty} W(r, \theta, s) r d r
$$

It should be noted that the phase distributions $P_{s}(\theta)$ can be negative for $s>-1$. Only the phase distributions obtained from the $Q$ function (or the smoothed $Q$ function)

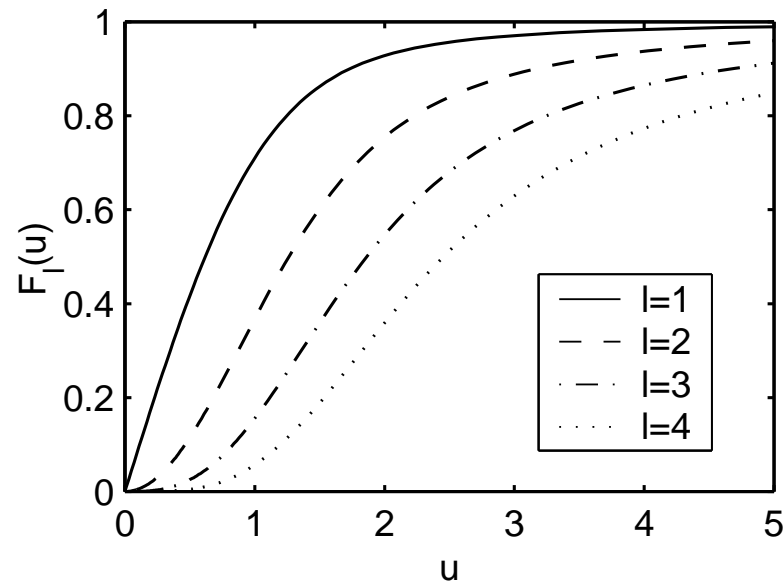

FIG. 1. Filtering functions $F_{l}(u)$ for determination of exponential phase moments $\Psi_{l}(s)$ from the $s_{0}$-parametrized quasidistributions.

are positively defined for every quantum state. Moreover, for $s>0$, the distributions can be highly singular generalized functions. Thus we restrict ourselves to the negative $s$ in the following.

The exponential phase moments are defined as

$$
\begin{aligned}
\Psi_{l}(s) & =\langle\exp (i l \theta)\rangle_{s}=\int P_{s}(\theta) e^{i l \theta} d \theta \\
& =\int_{0}^{2 \pi} \int_{0}^{\infty} W(r, \theta, s) e^{i l \theta} r d r d \theta .
\end{aligned}
$$

The moments $\Psi_{l}(s)$ can be simply determined from any quasidistribution $W\left(q, p, s_{0}\right)$ provided that $s_{0}>s$. Indeed, inserting the relation (2) into Eq. (41), we have

$$
\Psi_{l}(s)=\int_{0}^{\infty} \int_{0}^{2 \pi} F_{l}\left(\frac{r}{\sqrt{s_{0}-s}}\right) W\left(r, \theta, s_{0}\right) e^{i l \theta} r d r d \theta .
$$

The filtering functions $F_{l}(u)$ are given by

$$
F_{l}(u)=\frac{1}{\pi} \int_{0}^{\infty} \int_{0}^{2 \pi} e^{i l \phi} e^{-u^{2}-\rho^{2}+2 u \rho \cos \phi} \rho d \rho d \phi .
$$

Integration over the angle variable $\phi$ yields the modified Bessel function $I_{l}(2 u \rho)$. The resulting integral over radial variable $\rho$ can be found in the tables of integrals (Ref. [29], p. 306, Eq. 2.15.5.4) and we have

$$
F_{l}(u)=\sqrt{\pi} \frac{u}{2} \exp \left(-\frac{u^{2}}{2}\right)\left[I_{\frac{|l|-1}{2}}\left(\frac{u^{2}}{2}\right)+I_{\frac{|l|+1}{2}}\left(\frac{u^{2}}{2}\right)\right] .
$$

The first four filtering functions are plotted in Fig. 1. They start from zero and asymptotically reach unity. The interval, where the functions $F_{l}\left(r / \sqrt{s_{0}-s}\right)$ are significantly lower than 1 , increases with decreasing $s$. This implies that the absolute values of the phase moments 
$\Psi_{l}(s)$ decrease with decreasing $s$ because the modulation of the phase distribution $P_{s}(\theta)$ is suppressed by the smoothing convolution (2).

It is remarkable that the functions $F_{l}(u)$ are closely related to the exponential phase moments of the coherent state $|\xi\rangle$,

$$
\Psi_{l}(\xi ; s)=F_{l}\left(\sqrt{\frac{2}{1-s}}|\xi|\right) e^{i l \psi}, \quad \psi=\arg \xi
$$

To prove this, we notice that the quasidistributions $W_{s}(\alpha)$ of the coherent state $|\xi\rangle$ are shifted Gaussians,

$$
W(\alpha, s)=\frac{2}{(1-s) \pi} \exp \left(-\frac{2|\alpha-\xi|^{2}}{1-s}\right) .
$$

Inserting this into Eq. (4), we immediately obtain Eq. (8).

The filtering functions $F_{l}(u)$ can be expanded in Taylor series,

$$
F_{l}(u)=\sum_{n=0}^{\infty} f_{n, l} u^{2 n+|l|}
$$

where

$$
f_{n, l}=\frac{|l|}{2}(-1)^{n} \frac{\Gamma(n+|l| / 2)}{n !(n+|l|) !} .
$$

It is convenient to introduce the parameter $t, s_{0}-s=$ $1 / t^{2}$. With the help of the expansion (10), we can rewrite Eq. (5) as

$$
\begin{aligned}
& \Psi_{l}\left(s_{0}-\frac{1}{t^{2}}\right)= \\
& \quad \sum_{n=0}^{\infty} f_{n, l} \int_{0}^{\infty} \int_{0}^{2 \pi}(r t)^{2 n+|l|} W\left(r, \theta, s_{0}\right) e^{i l \theta} r d r d \theta .
\end{aligned}
$$

It follows from this formula that $\Psi_{l}(s)$ are generating functions of the $s_{0}$-ordered moments,

$$
\left\langle r^{2 n+|l|} e^{i l \theta}\right\rangle_{s_{0}}=\left.\frac{1}{(2 n+|l|) ! f_{n, l}} \frac{d^{2 n+|l|}}{d t^{2 n+|l|}} \Psi_{l}\left(s_{0}-\frac{1}{t^{2}}\right)\right|_{t=0}
$$

The limit $t \rightarrow 0$ should be taken only after the derivative is performed. The generating functions $\Psi_{l}(s)$ can be used to determine the moments $\left\langle r^{2 n+|l|} e^{i l \theta}\right\rangle_{s_{0}}$ for any ordering parameter $s_{0}$. Notice, however, that the formula (13) fails for $l=0$. The exponential phase moments do not allow us to determine the moments $\left\langle r^{2 n}\right\rangle$ which are related to photon-number statistics. As an example, consider the Fock state $|n\rangle$. This state is phase insensitive, $\Psi_{l}(s)=0$ for $l \neq 0$, and the phase is uniformly distributed over the $2 \pi$ interval, $P_{s}(\theta)=1 / 2 \pi$. Note also that the photon-number distribution $p(n)$ can be recovered from the phase-averaged quadrature distributions [30].
The $s$-ordered moments (13) are simply related to more familiar moments of creation and annihilation operators. With the help of $\alpha=2^{-1 / 2} r \exp (i \theta)$ we find that

$$
\left\langle a^{\dagger n} a^{n+l}\right\rangle_{s}=2^{-(n+l / 2)}\left\langle r^{2 n+l} e^{i l \theta}\right\rangle_{s}
$$

and a similar expression holds for $\left\langle a^{\dagger n+l} a^{n}\right\rangle_{s}$. The formula (13) allows us to find any moments $\left\langle a^{\dagger m} a^{n}\right\rangle$ provided that $m \neq n$. Complementarily, the moments $\left\langle a^{\dagger k} a^{k}\right\rangle=\left\langle: n^{k}:\right\rangle$ can be determined from the photonnumber distribution.

The phase moments $\Psi_{l}(s)$ are linear combinations of density-matrix elements $\rho_{n+l, n}$,

$$
\Psi_{l}(s)=\sum_{n=0}^{\infty} c_{n, l}(s) \rho_{n+l, n}
$$

where 32

$$
\begin{aligned}
c_{n, l}(s)= & \left(\frac{2}{1-s}\right)^{n+l / 2}[n !(n+l) !]^{1 / 2} \\
& \times \sum_{k=0}^{n} \frac{\Gamma(n-k+l / 2+1)}{k !(n-k) !(n+l-k) !}\left(-\frac{1+s}{2}\right)^{k} .
\end{aligned}
$$

If $l \neq 0$, the relation (15) can be inverted and $\rho_{n+l, n}$ can be found from $\Psi_{l}(s)$. In principle, the knowledge of $\Psi_{l}(s)$ at an infinite but countable number of points $s_{j}$ can be sufficient for determination of all $\rho_{n+l, n}$ from Eq. (15). Diagonal matrix elements appear only in

$$
\Psi_{0}(s)=\sum_{n=0}^{\infty} \rho_{n n} \equiv \operatorname{Tr} \rho=1,
$$

and this relation cannot be inverted. Only when we know both the phase moments $\Psi_{l}(s)$ and the photonnumber distribution $p(n)=\rho_{n n}$ can we determine all density-matrix elements $\rho_{m n}$ or, equivalently, all moments $\left\langle a^{\dagger n} a^{m}\right\rangle_{s}$. Thus the simultaneous knowledge of the functions $\Psi_{l}(s)$ and $p(n)$ provides complete information on the quantum state and it is equivalent to the knowledge of the Wigner function or the density matrix.

\section{SAMPLING KERNELS FOR THE EXPONENTIAL PHASE MOMENTS}

Balanced homodyne detection provides statistics $w\left(x_{\theta}, \theta\right)$ of rotated quadratures,

$$
x_{\theta}=\frac{1}{\sqrt{2}}\left(a e^{-i \theta}+a^{\dagger} e^{i \theta}\right),
$$

where $\theta$ is the relative phase between the $\mathrm{LO}$ and the signal mode. The probability distribution $w\left(x_{\theta}, \theta\right)$ can be obtained from the Wigner function $W(q, p)$ as a marginal distribution [6], 


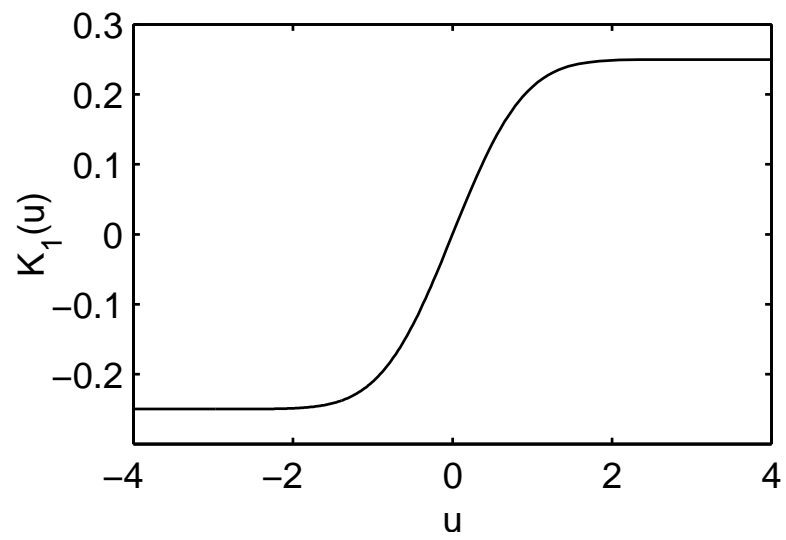

FIG. 2. Kernel $K_{1}(u)$ for sampling of odd exponential phase moments.

$$
\begin{aligned}
w\left(x_{\theta}, \theta\right)= & \int_{-\infty}^{\infty} \int_{-\infty}^{\infty} \delta\left(x_{\theta}-q \cos \theta-p \sin \theta\right) \\
& \times W(q, p) d q d p .
\end{aligned}
$$

We would like to sample the moments $\Psi_{l}(s)$ directly from the homodyne data $w\left(x_{\theta}, \theta\right)$ with the use of the kernels $\mathcal{K}_{l}\left(x_{\theta}, \theta ; s\right)$ :

$$
\Psi_{l}(s)=\int_{-\infty}^{\infty} \int_{0}^{2 \pi} \mathcal{K}_{l}\left(x_{\theta}, \theta ; s\right) w\left(x_{\theta}, \theta\right) d x_{\theta} d \theta .
$$

The $\theta$ dependence of the kernels must be of the form $\exp (i l \theta)$ [9]. Thus we look for the kernels in the form

$$
\mathcal{K}_{l}\left(x_{\theta}, \theta ; s\right)=K_{l}\left(x_{\theta}, s\right) e^{i l \theta} .
$$

In what follows we restrict ourselves to positive $l$. For negative $l$, the exponential moments can be obtained by complex conjugation, $\Psi_{-l}(s)=\Psi_{l}^{*}(s)$. Now we substitute Eq. (19) into Eq. (20), perform integration over $x_{\theta}$, and rewrite the remaining integral in polar coordinates. After some algebra, we arrive at

$$
\begin{aligned}
\Psi_{l}(s)= & \int_{0}^{2 \pi} \int_{0}^{\infty}\left[\int_{0}^{2 \pi} K_{l}(r \cos \phi, s) e^{i l \phi} d \phi\right] \\
& \times W(r, \theta) e^{i l \theta} r d r d \theta .
\end{aligned}
$$

Comparing the formulas (22) and (5), where we set $s_{0}=$ 0 , we conclude that the kernel $K_{l}\left(x_{\theta}, s\right)$ must fulfill the integral equation

$$
\int_{0}^{2 \pi} K_{l}(r \cos \theta, s) e^{i l \theta} d \theta=F_{l}\left(r /|s|^{1 / 2}\right) .
$$

In order to solve this equation, we expand the kernel $K_{l}\left(x_{\theta}, s\right)$ in Taylor series,

$$
K_{l}\left(x_{\theta}, s\right)=\sum_{n=0}^{\infty} a_{n}(l, s) x_{\theta}^{n} .
$$

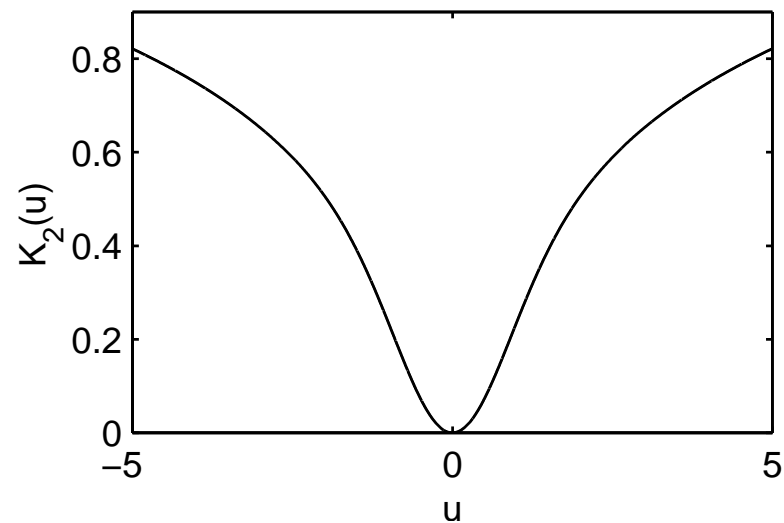

FIG. 3. Kernel $K_{2}(u)$ for sampling of even exponential phase moments.

This expansion is inserted into Eq. (23) and the integration over $\theta$ is carried out, using the formula

$$
\int_{0}^{2 \pi}(\cos \theta)^{2 n+l} e^{i l \theta} d \theta=\frac{2 \pi}{2^{2 n+l}}\left(\begin{array}{c}
2 n+l \\
n
\end{array}\right) .
$$

Comparing the Taylor series on the left-hand side of Eq. (23) with the series (10), we find the coefficients $a_{n}(l, s)$. Inserting them back into the series (24), we arrive at

$K_{l}\left(x_{\theta}, s\right)=\frac{l}{4 \pi} \sum_{n=0}^{\infty}(-1)^{n} \frac{\Gamma(n+l / 2)}{(2 n+l) !}\left(\frac{2 x_{\theta}}{|s|^{1 / 2}}\right)^{2 n+l}$.

Notice that the kernel is a function of a specific combination of $x_{\theta}$ and $s, u=x_{\theta} /|s|^{1 / 2}$. In the following we use $u$ for simplicity. Let us discuss the relation between the kernels $K_{l}(u)$ and $K_{l+2}(u)$. We have

$$
\begin{aligned}
K_{l+2}(u) & =-\frac{l+2}{4 \pi} \sum_{n=1}^{\infty}(-1)^{n} \frac{\Gamma(n+l / 2)}{(2 n+l) !}(2 u)^{2 n+l} \\
& =-\frac{l+2}{l} K_{l}(u)+\frac{l+2}{4 \pi} \frac{\Gamma(l / 2)}{l !}(2 u)^{l} .
\end{aligned}
$$

However, the kernels $K_{l}$ are not uniquely determined. Any polynomial of order lower than $l$ can be added to kernel $K_{l}$, because all such polynomials are solutions of the homogeneous integral equation

$$
\int_{0}^{2 \pi} f(r \cos \theta) e^{i l \theta}=0 .
$$

Thus we can neglect the last term in the formula (27) and we can define the kernels for which

$$
K_{l+2}(u)=-\frac{l+2}{l} K_{l}(u)
$$

holds. It remains to find out the kernels $K_{1}$ and $K_{2}$. The summation of the series can be found in the Appendix. The results are

$$
K_{1}(u)=\frac{1}{4} \operatorname{erf}(u),
$$




$$
K_{2}(u)=\frac{1}{\sqrt{\pi}} \int_{0}^{u} e^{-y^{2}} \operatorname{erfi}(y) d y .
$$

The kernels are plotted in Fig. 2 and Fig. 3, respectively.

Combining this result with the recurrence formula (29), we finally have

$$
\begin{aligned}
\mathcal{K}_{2 l+1}\left(x_{\theta}, \theta ; s\right) & =(-1)^{l}(2 l+1) K_{1}\left(x_{\theta} /|s|^{\frac{1}{2}}\right) e^{i(2 l+1) \theta} \\
\mathcal{K}_{2 l}\left(x_{\theta}, \theta ; s\right) & =(-1)^{l-1} l K_{2}\left(x_{\theta} /|s|^{\frac{1}{2}}\right) e^{i 2 l \theta}
\end{aligned}
$$

For large $x_{\theta}$, all the kernels tend to the same limit because we move to the strong classical field domain and the differences between various $s$ orderings vanish. The limit for odd kernels is straightforward. We simply notice that

$$
\lim _{x \rightarrow \pm \infty} \operatorname{erf}(x)= \pm 1
$$

The limit for even kernels can be found if we take into account that for large $x$,

$$
e^{-x^{2}} \operatorname{erfi}(x) \approx \frac{1}{\sqrt{\pi}} \frac{1}{x} .
$$

Inserting this into Eq. (32) we have for large $x_{\theta}$

$$
\mathcal{K}_{2 l}\left(x_{\theta}, \theta ; s\right) \approx \frac{1}{\pi} l(-1)^{l-1} \ln \left|x_{\theta}\right| e^{i 2 l \theta}+C_{l, s} e^{i 2 l \theta} .
$$

Here $C_{l, s}$ is some constant. The superfluous term containing this constant can be omitted for reasons discussed above and we can see that as a limit all kernels approach those for the phase moments of the Wigner function [9]:

$$
\begin{aligned}
\mathcal{K}_{2 l+1} & =\frac{1}{4}(2 l+1)(-1)^{l} \operatorname{sgn}\left(x_{\theta}\right) e^{i(2 l+1) \theta}, \\
\mathcal{K}_{2 l} & =\frac{1}{\pi} l(-1)^{l-1} \ln \left|x_{\theta}\right| e^{i 2 l \theta} .
\end{aligned}
$$

Up to now, we have considered ideal detectors having unit quantum efficiency. In a realistic experiment, the detection efficiency $\eta$ is lower than $100 \%$ and the smoothed quadrature distributions $w\left(x_{\theta}, \theta ; \eta\right)$ are recorded [31],

$$
\begin{aligned}
w\left(x_{\theta}, \theta ; \eta\right)= & \frac{1}{\sqrt{\pi(1-\eta)}} \\
& \times \int_{-\infty}^{\infty} w\left(x_{\theta}^{\prime}, \theta\right) \exp \left[-\frac{\left(x_{\theta}-\sqrt{\eta} x_{\theta}^{\prime}\right)^{2}}{1-\eta}\right] d x_{\theta}^{\prime} .
\end{aligned}
$$

The smoothed quadrature distributions $w\left(x_{\theta}, \theta ; \eta\right)$ can be obtained from the scaled and smoothed Wigner function,

$$
\begin{aligned}
w\left(x_{\theta}, \theta ; \eta\right)= & \int_{-\infty}^{\infty} \int_{-\infty}^{\infty} \delta\left(x_{\theta}-q \cos \theta-p \sin \theta\right) \\
& \times \frac{1}{\eta} W\left(\frac{q}{\sqrt{\eta}}, \frac{p}{\sqrt{\eta}},-\frac{1-\eta}{\eta}\right) d q d p .
\end{aligned}
$$
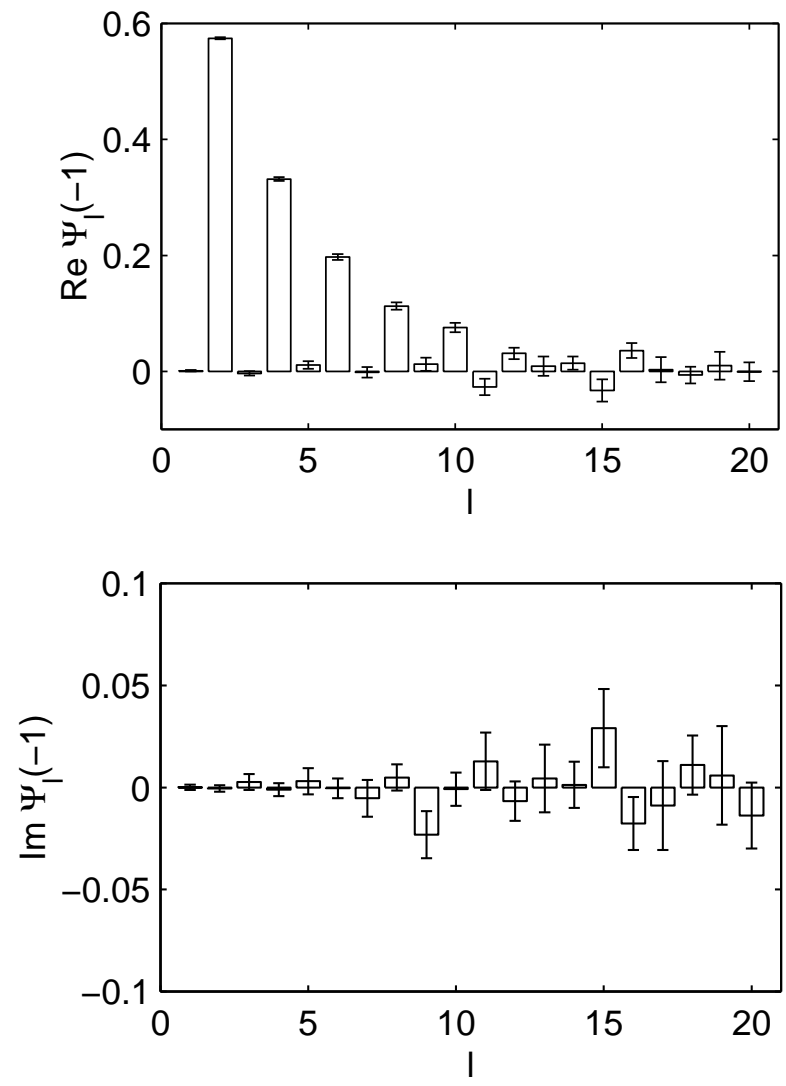

FIG. 4. Reconstructed phase moments $\Psi_{l}(-1)$ of squeezed vacuum state $|\zeta\rangle, \zeta=1.317$, i.e. $\langle n\rangle=3$. Statistical errors are denoted by error bars.

The scaling and smoothing are two factors which must be included in the kernels $\mathcal{K}_{l}\left(x_{\theta}, \theta ; s, \eta\right)$. The scaling means that we must replace $x_{\theta}$ by $x_{\theta} / \sqrt{\eta}$. The smoothing tells us that the kernels $\mathcal{K}\left(x_{\theta}, \theta, s\right)$ would provide us with exponential phase moments $\Psi_{l}\left(s+s_{\eta}\right), s_{\eta}=-(1-\eta) / \eta$. Thus we must replace $s$ with $s-s_{\eta}$ in all the expressions (32). It is obvious that the losses impose a new limit. We can reconstruct only exponential phase moments for the phase distributions corresponding to $s<s_{\eta}$. The modified kernels are

$$
\mathcal{K}_{l}\left(x_{\theta}, \theta ; s, \eta\right)=\mathcal{K}_{l}\left(x_{\theta} / \sqrt{\eta}, \theta ; s+(1-\eta) / \eta\right),
$$

and the condition $s<-(1-\eta) / \eta$ must be fulfilled.

\section{MONTE CARLO SIMULATIONS}

In order to test the kernels, we performed Monte Carlo simulations of the homodyne detection and we present here the results of simulations for the squeezed vacuum state $|\zeta\rangle$,

$$
|\zeta\rangle=\exp \left(\frac{1}{2} \zeta a^{\dagger 2}-\frac{1}{2} \zeta^{*} a^{2}\right)|0\rangle,
$$




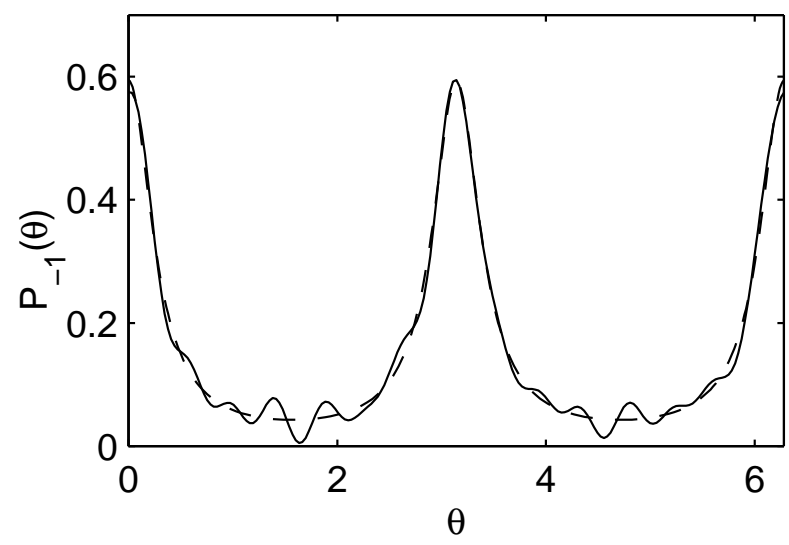

FIG. 5. Reconstruction of the phase distribution $P_{-1}(\theta)$ of the squeezed vacuum state from the sampled exponential moments depicted in Fig. 4. The solid line shows the reconstructed distribution and the dashed line represents the exact shape.

where $|0\rangle$ is the vacuum state. The squeezed vacuum state belongs to the class of Gaussian states, i.e. states whose quasidistributions $W(q, p, s)$ have Gaussian form. The phase distribution $P_{s}(\theta)$ for the general Gaussian mixed state was determined in 32,33. In particular, it holds that $P_{s}(\theta)$ of the squeezed vacuum state can be expressed as [33]

$$
P_{s}(\theta)=\frac{1}{2 \pi} \frac{\left(B_{s}^{2}-C^{2}\right)^{1 / 2}}{B_{s}-C \cos (2 \theta-\psi)}
$$

where

$$
\begin{aligned}
B_{s} & =\sinh ^{2}|\zeta|+(1-s) / 2, \\
C & =\frac{1}{2} \sinh (2|\zeta|),
\end{aligned}
$$

and $\psi=\arg \zeta$. The phase moments can be calculated with the help of the residue theorem. One arrives at

$$
\begin{aligned}
\Psi_{2 l}(s) & =\left(B_{s} / C-\sqrt{B_{s}^{2} / C^{2}-1}\right)^{l} e^{i l \psi}, \\
\Psi_{2 l-1}(s) & =0 .
\end{aligned}
$$

In our simulations, the sampling was performed for 120 values of $\theta$ equidistantly placed at the interval $[0,2 \pi]$ and 5000 samples have been made for each $\theta$. We assumed that the overall detection efficiency is $\eta=80 \%$ and we used the loss compensating kernels (39).

Figure 4 shows the reconstructed phase moments of the $Q$ function, $\Psi_{l}(-1)$. The results are in very good agreement with the exact values following from Eq. (43). Statistical errors were calculated in a manner described in [9]. As a rule, error increases with increasing $l$ and this uncertainty is responsible for the fast oscillations in the reconstructed probability distribution $P_{-1}(\theta)$, see Fig. 5 .

The reconstructed moments $\Psi_{l}(s)$, considered as functions of the ordering parameter $s$, are plotted in Fig. 6 .

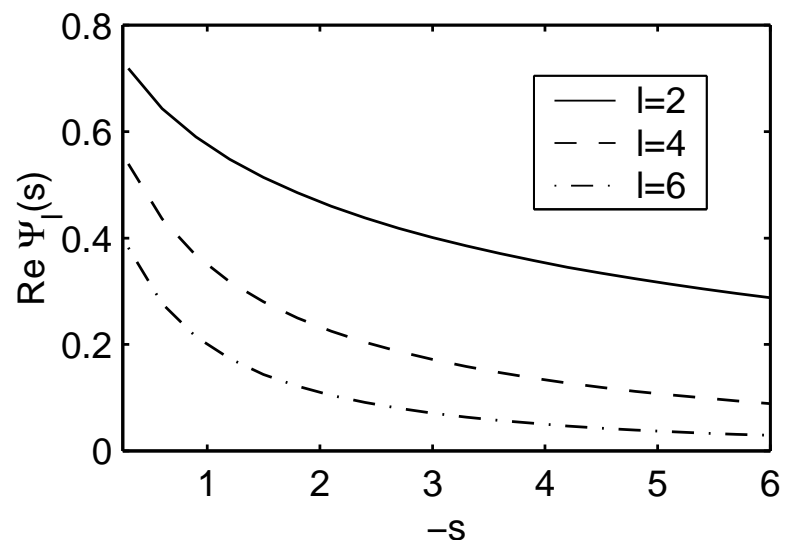

FIG. 6. Reconstructed phase moments $\Psi_{l}(s)$ as functions of the $s$ parameter.

Again, we found that the curves are in good agreement with their theoretical counterparts. Notice that, due to the assumed $80 \%$ efficiency of the detection, we were able to sample only moments for $s<-0.25$.

We repeated our simulations also for other types of quantum states such as coherent states and displaced Fock states. In all cases, the reconstruction procedure worked well and the sampled moments were in good agreement with the theoretical values. We emphasize that we have used only $6 \times 10^{5}$ samples in our simulations and such an amount of data can be routinely recorded in the experiment.

\section{CONCLUSIONS}

We have shown that the exponential phase moments of the $s$-parametrized quasidistributions are generating functions of the moments of creation and annihilation operators. A simultaneous knowledge of photon-number distribution and the functions $\Psi_{l}(s)$ provides a complete description of the quantum state. We have found kernels for direct sampling of the moments $\Psi_{l}(s)$ from quadrature distributions measured in optical homodyne detection. The detection efficiency $\eta$ imposes a bound on the ordering parameter, we can sample only phase moments for $s<-(1-\eta) / \eta$. In the ideal case $\eta=1$ and the Wigner function represents the limit; for $\eta=0.5$ the limit is formed by a $Q$ function. We performed numerical Monte Carlo simulations of homodyne detection, thereby demonstrating the feasibility of direct sampling of the exponential phase moments from experimental data.

\section{ACKNOWLEDGMENTS}

I would like to thank T. Opatrný, J. Peřina, and I.Sh. Averbukh for helpful discussions. I am pleased to acknowledge support of the U.S.-Israel Binational Science Foundation (Grant No. 96-00432). 


\section{APPENDIX: SUMMATION OF THE SERIES FOR THE KERNELS $K_{1}$ AND $K_{2}$}

Here we sum the Taylor series for kernels $K_{1}(u)$ and $K_{2}(u)$. We start with $K_{1}(u)$. Using the formula for the Gamma function of a half-integer,

$$
\Gamma(n+1 / 2)=\sqrt{\pi} \frac{(2 n) !}{2^{2 n} n !},
$$

the series for $K_{1}(u)$ take on the form

$$
K_{1}(u)=\frac{1}{4 \pi} \sum_{n=0}^{\infty}(-1)^{n} \frac{2 \sqrt{\pi}}{n !} \frac{u^{2 n+1}}{(2 n+1)} .
$$

The derivative of the kernel is

$$
\frac{d}{d u} K_{1}(u)=\frac{1}{4} \sum_{n=0}^{\infty}(-1)^{n} \frac{2}{\sqrt{\pi}} \frac{u^{2 n}}{n !}=\frac{1}{4} \frac{2}{\sqrt{\pi}} e^{-u^{2}} .
$$

Integrating the above equation we arrive at

$$
K_{1}(u)=\frac{1}{4} \operatorname{erf}(u)
$$

We adopt a similar approach to determine $K_{2}(u)$,

$$
K_{2}(u)=\frac{1}{2 \pi} \sum_{n=0}^{\infty}(-1)^{n} \frac{n !}{(2 n+2) !}(2 u)^{2 n+2} .
$$

We calculate the derivatives

$$
f(u)=\frac{d}{d u} K_{2}(u)=\frac{2}{2 \pi} \sum_{n=0}^{\infty}(-1)^{n} \frac{n !}{(2 n+1) !}(2 u)^{2 n+1}
$$

and

$$
\begin{aligned}
f^{\prime}(u) & =\frac{d^{2}}{d u^{2}} K_{2}(u)=\frac{4}{2 \pi} \sum_{n=0}^{\infty}(-1)^{n} \frac{n !}{(2 n) !}(2 u)^{2 n} \\
& =\frac{2}{2 \pi} \sum_{n=1}^{\infty}(-1)^{n} \frac{(n-1) !}{(2 n-1) !}(2 u)^{2 n}+\frac{2}{\pi} .
\end{aligned}
$$

Thus we have

$$
f^{\prime}(u)=-2 u f(u)+\frac{2}{\pi}
$$

Let us look for the function $f(u)$ in the form

$$
f(u)=\frac{2}{\pi} \frac{g(u)}{g^{\prime}(u)} .
$$

Substituting this into the above equation, we finish with

$$
\begin{aligned}
g^{\prime \prime}(u) & =2 u g^{\prime}(u), \\
g(u) & =\frac{\sqrt{\pi}}{2} \operatorname{erfi}(u) .
\end{aligned}
$$

Inserting this into Eq. (A9), we conclude that

$$
K_{2}(u)=\frac{1}{\sqrt{\pi}} \int_{0}^{u} e^{-y^{2}} \operatorname{erfi}(y) d y .
$$

[1] D.T. Smithey, M. Beck, M.G. Raymer, and A. Faridani, Phys. Rev. Lett. 70, 1244 (1993).

[2] G. Breitenbach, T. Müller, S.F. Pereira. J.-Ph. Poizat, S. Schiller, and J. Mlynek, J. Opt. Soc. Am. B 12, 2304 (1995); S. Schiller, G. Breitenbach, S.F. Pereira, T. Müller, and J. Mlynek, Phys. Rev. Lett. 77, 2933 (1996).

[3] T.J. Dunn, I.A. Walmsley, and S. Mukamel, Phys. Rev. Lett. 74, 884 (1995).

[4] S. Wallentowitz and W. Vogel, Phys. Rev. Lett. 75, 2932 (1995).

[5] D. Leibfried, D.M. Meekhof, B.E. King, C. Monroe, W.M. Itano, and D.J. Wineland, Phys. Rev. Lett. 77, 4281 (1996).

[6] K. Vogel and H. Risken, Phys. Rev. A 40, 2847 (1989).

[7] G.M. D'Ariano, C. Macchiavello, and M.G.A. Paris, Phys. Rev. A 50, 4298 (1994); U. Leonhardt, H. Paul, and G.M. D'Ariano, ibid. 52, 4899 (1995); Th. Richter, Phys. Lett. A 211, 327 (1996); U. Leonhardt, M. Munroe, T. Kiss, Th. Richter, and M.G. Raymer, Opt. Commun. 127, 144 (1996).

[8] Th. Richter, Phys. Rev. A 53, 1197 (1996); A. Wünsche, ibid. 54, 5291 (1996).

[9] M. Dakna, T. Opatrný, and D.G. Welsch, Opt. Commun. 148, 355 (1998); M. Dakna, G. Breitenbach, J. Mlynek, T. Opatrný, S. Schiller, and D.G. Welsch, ibid. 152, 289 (1998).

[10] Z. Kis, T. Kiss, J. Janszky, P. Adam, S. Wallentowitz, and W. Vogel, Phys. Rev. A 59, R39 (1999); Th. Richter, J. Opt. B: Quantum Semiclass. Opt. 1, 650 (1999).

[11] Z. Hradil, Phys. Rev. A 55, R1561 (1997); Z. Hradil, J. Summhammer, and H. Rauch, Phys. Lett. A 261, 20 (1999).

[12] K. Banaszek, Phys. Rev. A 57, 5013 (1998).

[13] K. Banaszek, G.M. D'Ariano, M.G.A. Paris, and M.F. Sacchi, Phys. Rev. A 61, R010304 (1999).

[14] D.G. Welsch, W. Vogel, and T. Opatrný, in Homodyne Detection and Quantum-state Reconstruction, Progress in Optics Vol. 39, edited by E. Wolf (Elsevier, Amsterdam, 1999).

[15] F. London, Z. Phys. 40, 193 (1927).

[16] D.T. Pegg and S.M. Barnett, Europhys. Lett. 6, 483 (1989); Phys. Rev. A 39, 1665 (1989); J. Mod. Opt. 36, 7 (1989).

[17] M.G.A. Paris, Phys. Rev. A 60, 5136 (1999).

[18] W. Schleich, R.J. Horowicz, and S. Varro, Phys. Rev. A 40, 7405 (1989).

[19] S.L. Braunstein and C.M. Caves, Phys. Rev. A 42, 4115 (1990).

[20] R. Tanaś, A. Miranowicz, and Ts. Gantsog, Quantum Phase Properties of Nonlinear Optical Phenomena, 
Progress in Optics, Vol. 35, Ed.: E. Wolf, Elsevier: Amsterdam, 1996.

[21] W. Schleich, A. Bandilla, and H. Paul, Phys. Rev. A 45, 6652 (1992).

[22] U. Leonhardt and H. Paul, Phys. Rev. A 47, 2460 (1993).

[23] J.W. Noh, A. Fougères, and L. Mandel, Phys. Rev. Lett. 67, 1426 (1991); Phys. Rev. A 45, 424 (1992); Phys. Rev. A 46, 2840 (1992).

[24] U. Leonhardt, J.A. Vaccaro, B. Böhmer, and H. Paul, Phys. Rev. A 51, 84 (1995).

[25] V. Peřinová, A. Lukš, and J. Peřina, Phase in Optics, World Scientific, Singapore, 1998.

[26] M. Beck, D.T. Smithey, and M.G. Raymer, Phys. Rev. A 48, R890 (1993); D.T. Smithey, M. Beck, J. Cooper, and M.G. Raymer, ibid. 48, 3159 (1993).

[27] T. Opatrný, M. Dakna, and D.G. Welsch, Phys. Rev. A 57, 2129 (1998).

[28] J. Peřina, Quantum Statistics of Linear and Nonlinear Optical Phenomena, (Kluwer, Dordrecht, 1991).

[29] A.P. Prudnikov, Y.A. Brychkov, and O.I. Marichev, Integrals and series, Vol. 2: Special functions, (Overseas Publishers Association, Amsterdam, 1998).

[30] M. Munroe, D. Boggavarapu, M.E. Anderson, and M.G. Raymer, Phys. Rev. A 52, R924 (1995).

[31] W. Vogel and J. Grabow, Phys. Rev. A 47, 4227 (1993).

[32] R. Tanaś, A. Miranowicz, and Ts. Gantsog, Phys. Scr. T48, 53 (1993).

[33] L. Mišta Jr., J. Řeháček, and J. Peřina, J. Mod. Opt. 45, 2269 (1998). 